\title{
Local convergence of quasi-Newton methods under metric regularity
}

\author{
F. J. Aragón Artacho* A. Belyakov ${ }^{\dagger}$ A. L. Dontchev ${ }^{\ddagger}$ M. López ${ }^{\S}$
}

July 25, 2013

\begin{abstract}
We consider quasi-Newton methods for generalized equations in Banach spaces under metric regularity and give a sufficient condition for q-linear convergence. Then we show that the well-known Broyden update satisfies this sufficient condition in Hilbert spaces. We also establish various modes of q-superlinear convergence of the Broyden update under strong metric subregularity, metric regularity and strong metric regularity. Simple numerical examples illustrate the results.
\end{abstract}

Keywords. Generalized equation, quasi-Newton method, Broyden update, strong metric subregularity, metric regularity, strong metric regularity, q-superlinear convergence.

AMS Subject Classification (2010). Primary: 49J53, Secondary: 49K40, 65H20, 90C30.

*Centre for Computer Assisted Research Mathematics and its Applications (CARMA), University of Newcastle, Callaghan, NSW 2308, Australia. Partially supported by various Australian Research Council grants.

${ }^{\dagger}$ Institute of Mathematical Methods in Economics, Vienna University of Technology, A-1040 Vienna, Austria. Supported by the Austrian Science Foundation (FWF) under grant No P 24125-N13. On leave from Institute of Mechanics, Lomonosov Moscow State University, 119192, Russia

${ }^{\ddagger}$ Mathematical Reviews, Ann Arbor, MI 48107-8604. Supported by NSF Grant DMS 1008341 through the University of Michigan.

${ }^{\S}$ Department of Statistics and Operations Research, University of Alicante, 03080 Alicante, Spain. Supported by MINECO of Spain, Grant MTM2011-29064-C03-02. 


\section{Introduction}

In this paper we consider the generalized equation

$$
f(x)+F(x) \ni 0,
$$

where $f: X \rightarrow Y$ is a function and $F: X \rightrightarrows Y$ is a set-valued mapping. Throughout, unless stated otherwise, $X$ and $Y$ are (real) Banach spaces. To simplify some of the arguments used we make the standing assumption that $f$ is continuously Fréchet differentiable everywhere with derivative $D f$ and $F$ has closed graph.

If $F$ is the zero mapping, then (1) reduces to the equation $f(x)=0$ for which the standard Newton iteration takes the form

$$
f\left(x_{k}\right)+D f\left(x_{k}\right)\left(x_{k+1}-x_{k}\right)=0 .
$$

When $F$ is nonzero, then the Newton iteration is extended in a natural way to

$$
f\left(x_{k}\right)+D f\left(x_{k}\right)\left(x_{k+1}-x_{k}\right)+F\left(x_{k+1}\right) \ni 0,
$$

that is, at each iteration we solve a partially linearized inclusion. In a path-breaking work N. H. Josephy [16] was the first to consider a Newton iteration of the kind (2) specialized to the case where $F$ is the normal cone mapping; then (1) describes a variational inequality. Most importantly, he employed the property of strong regularity coined by his $\mathrm{PhD}$ advisor S. M. Robinson [21]. In this paper, we adopt the definition given in [11]:

Definition 1.1 (strong metric regularity). A mapping $H: X \rightrightarrows Y$ is said to be strongly metrically regular at $\bar{x}$ for $\bar{y}$ when $\bar{y} \in H(\bar{x})$ and there are neighborhoods $U$ of $\bar{x}$ and $V$ of $\bar{y}$ such that the mapping $y \mapsto H^{-1}(y) \cap U$ is a Lipschitz continuous function on $V$.

The result of Josephy [16] adapted for the generalized equation (1) essentially says that that if $\bar{x}$ is a solution of (1), the function $f$ is twice continuously differentiable around $\bar{x}$ and the mapping $f+F$ is strongly metrically regular at $\bar{x}$ for 0 , then there exists a neighborhood $O$ of $\bar{x}$ such that for any starting point $x_{0} \in O$ the iteration (2) generates a unique sequence in $O$ and this sequence is q-quadratically convergent to $\bar{x}$.

A linear and bounded mapping $A: X \rightarrow Y$ is strongly metrically regular (everywhere) whenever its inverse $A^{-1}$ is single-valued. If the mapping $A$ is not necessarily invertible but only surjective, then it is metrically regular. Metric regularity has played a major role in nonlinear analysis in the last century, but whose importance has been fully recognized only recently. Its formal definition follows:

Definition 1.2 (metric regularity). A mapping $H: X \rightrightarrows Y$ is said to be metrically regular at $\bar{x}$ for $\bar{y}$ when $\bar{y} \in H(\bar{x})$ and there is a constant $\kappa>0$ together with neighborhoods $U$ of $\bar{x}$ and $V$ of $\bar{y}$ such that

$$
d\left(x, H^{-1}(y)\right) \leq \kappa d(y, H(x)) \quad \text { for all } \quad(x, y) \in U \times V .
$$

A central result in the theory of metric regularity is the Lyusternik-Graves theorem which says that if a function $f: X \rightarrow Y$ is continuously Fréchet differentiable around $\bar{x}$, then it is metrically regular at $\bar{x}$ (for $f(\bar{x})$ ) if and only if the derivative $D f(\bar{x})$ is surjective. In parallel, the standard 
inverse function theorem can be stated as follows: if a function $f: X \rightarrow Y$ is continuously Fréchet differentiable around $\bar{x}$ then it is strongly metrically regular at $\bar{x}$ (for $f(\bar{x})$ ) if and only if the derivative $D f(\bar{x})$ is invertible. The inverse function theorem can be extended in a natural way to the generalized equation (1): the mapping $f+F$ is (strongly) metrically regular at $\bar{x}$ for 0 if and only if the "partial linearization" $f(\bar{x})+D f(\bar{x})(\cdot-\bar{x})+F(\cdot)$ has the same property. This general pattern culminates in the inverse function theorem paradigm to which the recent book [11] is dedicated.

The third author of the present paper proved in [7], see also Theorem 6C.6 in [11], the following result which complements that of Josephy: if the derivative $D f$ is Lipschitz continuous around $\bar{x}$ and the mapping $f+F$ is metrically regular at $\bar{x}$ for 0 , then there exists a neighborhood $O$ of $\bar{x}$ such that for any starting point $x_{0} \in O$ there exists a sequence generated by (2) which stays in $O$ and this sequence is q-quadratically convergent to $\bar{x}$. By no means this sequence is to be unique, as in Josephy's theorem. Still the result in [7] has opened the way to developing a broader perspective to set-valued extensions of Newton's method. A number of results in this direction are presented in the books [1], [11] and [19].

There is a third regularity property that plays an important role in establishing convergence of Newton's method.

Definition 1.3 (strong metric subregularity). Consider a mapping $H: X \rightrightarrows Y$ and a point $(\bar{x}, \bar{y}) \in X \times Y$. Then $H$ is said to be strongly metrically subregular at $\bar{x}$ for $\bar{y}$ when $\bar{y} \in H(\bar{x})$ and there is a constant $\kappa>0$ together with neighborhoods $U$ of $\bar{x}$ such that

$$
\|x-\bar{x}\| \leq \kappa d(\bar{y}, H(x)) \text { for all } x \in U .
$$

Strong metric subregularity of $H$ at $\bar{x}$ for $\bar{y}$ implies that $\bar{x}$ is an isolated point in $H^{-1}(\bar{y})$; moreover, it is equivalent to the so-called isolated calmness of the inverse $H^{-1}$, meaning that there is a neighborhood $U$ of $\bar{x}$ such that $H^{-1}(y) \cap U \subset \bar{x}+\kappa\|y-\bar{x}\| \mathbb{B}$ for all $y \in Y$. The isolated calmness was introduced independently in [4] under the name semistability and in [6] under the name local upper Lipschitz continuity. Every mapping $H$ acting in finite dimensions, whose graph is the union of finitely many convex polyhedral sets, is strongly metrically subregular at $\bar{x}$ for $\bar{y}$ if and only if $\bar{x}$ is an isolated point in $H^{-1}(\bar{y})$. Most importantly, the strong metric subregularity obeys the paradigm of the inverse function theorem in the same way as metric regularity and strong metric regularity do. In particular, a smooth function $f$ is strongly metrically subregular at $\bar{x}$ if and only if its derivative mapping $D f(\bar{x})$ is injective. Consider the Newton method (2) for the generalized equation (1) with a function $f$ whose derivative mapping $D f$ is Lipschitz continuous around a reference solution $\bar{x}$. If a sequence $\left\{x_{k}\right\}$ generated by (2) is convergent to $\bar{x}$ then, as shown in [4, Corollary 2.1], strong metric subregularity implies that this sequence converges quadratically. Actually, in this case the following sharper result is shown in [8], see also [11, Section 6c]: there exists a neighborhood $O$ of $\bar{x}$ such that if a sequence $\left\{x_{k}\right\}$ generated by (2) stays in $O$ for all $k$, then it is quadratically convergence to $\bar{x}$. Note that the strong metric subregularity itself does not guarantee the existence of a Newton sequence. In order to ensure that Newton's method (2) is executable, the strong metric subregularity is usually combined with Bonnans [4] introduced a property called by him hemistability which basically postulates the existence of a Newton iteration. Specifically, in our notation this property requires that for ant $\varepsilon>0$ there exists $\delta>0$ such that for any $x$ and $M$ with $\|x-\bar{x}\|+\|M-D f(\bar{x})\| \leq \varepsilon$ there exists $\hat{x}$ with $\|\hat{x}-\bar{x}\| \leq \delta$ satisfying $f(x)+M(\hat{x}-x)+F(\hat{x})$. In order to handle Newton's method, among 
others, Klatte and Kummer, see [19] as well as previous papers of these authors, introduced the property that there exist neighborhoods $U$ of $\bar{x}$ and $V$ of 0 such that $(f+F)^{-1}(y) \cap U \neq \emptyset$ for all $y \in V$; then they called the combination of the strong metric subregularity and this property upper regularity. Observe that for the mapping $f+F$ with a smooth function $f$ hemistability is implied by metric regularity; this is a simple consequence of the Lyusternik-Graves theorem. The property introduced by Klatte and Kummer is also implied by metric regularity, see [11, Proposition 3E.1]. On the other hand the combination of semistability and hemistability is stronger than metric regularity, since metric regularity does not imply local uniqueness of the reference point, but is weaker than strong metric regularity.

In this paper we prove first a result parallel to the one in [7] concerning the convergence of the following quasi-Newton method: given $x_{0}$ compute $x_{k+1}$ to satisfy

$$
f\left(x_{k}\right)+B_{k}\left(x_{k+1}-x_{k}\right)+F\left(x_{k+1}\right) \ni 0, \quad \text { for } \quad k=0,1, \ldots,
$$

where $B_{k}$ is a sequence of linear and bounded mappings from $X$ to $Y$. The specific way $B_{k}$ is constructed determines the quasi-Newton method, e.g., Broyden, BFGS, SR1, etc. In Theorem 3.1, we show that if the mapping $f+F$ of (1) is metrically regular at $\bar{x}$ for 0 and the initial mapping $B_{0}$ is close to $D f(\bar{x})$, then, under certain condition on the sequence of mappings $B_{k}$, there exists a neighborhood $O$ of $\bar{x}$ such that for any starting point $x_{0} \in O$ there exists a sequence generated by (4) which stays in $O$ and is q-linearly convergent to $\bar{x}$. If in addition the mapping $f+F$ is strongly metrically regular, then there exists a unique in $O$ sequence generated by (4), and hence this sequence is q-linearly convergent.

In Section 4 we first prove in the Hilbert space setting that the Broyden method satisfies the condition stated in Theorem 3.1 and hence, under (strong) metric regularity, this method generates a (unique) sequence which is q-linearly convergent to the reference solution $\bar{x}$. In Theorem 4.9, on the condition that $B_{0}-D f(\bar{x})$ is a Hilbert-Schmidt operator, we establish q-superlinear convergence in three cases depending on the property of the mapping $f+F$ : (i) under strong metric subregularity there exists a neighborhood $O$ of $\bar{x}$ such that every sequence generated by (4) which stays in $O$ is q-superlinearly convergent to $\bar{x}$; (ii) if in addition $f+F$ is metrically regular then there exists a neighborhood $O$ of $\bar{x}$ such that for every starting point $x_{0}$ there exists a sequence generated by (4) which is q-superlinearly convergent to $\bar{x}$; (iii) under strong metric regularity the unique in $O$ sequence generated by (4) is q-superlinearly convergent to $\bar{x}$. A key step in proving this result, besides Theorem 3.1, is Proposition 4.8, where we show that in the case considered the Broyden update satisfies the Dennis-Moré condition, which allows us to employ the generalization of the Dennis-Moré theorem obtained in [9]. Theorem 4.9 sharpens, in the setting of (strong) metric (sub)regularity, and extends to infinite dimensions the results in [4] developed for variational inequalities in finite dimensions. Specifically, for a class of quasi-Newton methods including the Broyden update [4, Theorem 2.3] shows the existence of a q-superlinear convergent sequence under hemistability and semistability of the reference solution. In this paper we focus on the Broyden method extending, among other results, [4, Theorem 2.3] to infinite dimensions under the stronger condition that both metric regularity and strong metric subregularity hold.

Section 2 contains an auxiliary result concerning perturbed metric regularity, which is used as a tool for proving local convergence of the method (4). In Section 5 we present two numerical examples, the second of which is based on a model of economic equilibrium recently developed in [12]. 
We introduce next the notation, definitions and terminology we use throughout the paper, and also put our results in perspective to the previous developments on the subject. Any norm is denoted by $\|\cdot\|$ and any metric by $\rho(\cdot, \cdot)$. The distance from a point $x$ to a set $C$ is denoted by $d(x, C)$ and the excess from a set $D$ to a set $C$ by $e(D, C)=\sup _{x \in D} d(x, C)$. The closed ball centered at $x$ with radius $a$ is denoted by $\mathbb{B}_{a}(\bar{x})$ and $\mathcal{L}(X, Y)$ denotes the Banach space of linear and bounded mappings acting from $X$ to $Y$.

\section{Preliminaries}

We utilize the following generalization of Nadler's fixed point theorem, originally proved in [10], for more see [11, Theorem 5E.2]):

Theorem 2.1 (contraction mapping principle). Let $(X, \rho)$ be a complete metric space, and consider a set-valued mapping $\Phi: X \rightrightarrows X$, a point $\bar{x} \in X$, and positive scalars $a$ and $\theta$ such that $\theta<1$, the set $\operatorname{gph} \Phi \cap\left(\mathbb{B}_{a}(\bar{x}) \times \mathbb{B}_{a}(\bar{x})\right)$ is closed, and the following conditions hold:

(i) $d(\bar{x}, \Phi(\bar{x}))<a(1-\theta)$;

(ii) $e\left(\Phi(u) \cap \mathbb{B}_{a}(\bar{x}), \Phi(v)\right) \leq \theta \rho(u, v)$ for all $u, v \in \mathbb{B}_{a}(\bar{x})$.

Then $\Phi$ has a fixed point in $\mathbb{B}_{a}(\bar{x})$; that is, there exists $x \in \mathbb{B}_{a}(\bar{x})$ such that $x \in \Phi(x)$. In addition, if $\Phi$ is single-valued, then $\Phi$ has a unique fixed point in $\mathbb{B}_{a}(\bar{x})$.

Theorem 2.2 (perturbed metric regularity). Let $(X, \rho)$ be a complete metric space and $(Y, \rho)$ be a linear metric space with shift-invariant metric. Consider a mapping $H: X \rightrightarrows Y$ with closed graph and a point $(\bar{x}, \bar{y}) \in$ gph $H$ at which $H$ is metrically regular, that is, there exist positive constants $a, b$, and $\kappa$ such that

$$
d\left(x, H^{-1}(y)\right) \leq \kappa d(y, H(x)) \quad \text { for all } \quad(x, y) \in \mathbb{B}_{a}(\bar{x}) \times \mathbb{B}_{b}(\bar{y}) .
$$

Let $\mu>0$ be such that $\kappa \mu<1$ and let $\kappa^{\prime}>\kappa$. Then for every positive $\alpha$ and $\beta$ such that

$$
\alpha \leq a / 2, \quad \mu \alpha+2 \beta \leq b \quad \text { and } \quad 2 \kappa^{\prime} \beta \leq \alpha(1-\kappa \mu)
$$

and for every function $h: X \rightarrow Y$ satisfying

$$
\rho(h(\bar{x}), 0) \leq \beta
$$

and

$$
\rho\left(h(x), h\left(x^{\prime}\right)\right) \leq \mu \rho\left(x, x^{\prime}\right) \quad \text { for every } \quad x, x^{\prime} \in \mathbb{B}_{\alpha}(\bar{x}),
$$

the mapping $h+H$ has the following property: for every $y, y^{\prime} \in \mathbb{B}_{\beta}(\bar{y})$ and every $x \in(h+H)^{-1}(y) \cap$ $\mathbb{B}_{\alpha}(\bar{x})$ there exists $x^{\prime} \in(h+H)^{-1}\left(y^{\prime}\right)$ such that

$$
\rho\left(x, x^{\prime}\right) \leq \frac{\kappa^{\prime}}{1-\kappa \mu} \rho\left(y, y^{\prime}\right) .
$$

In addition, if the mapping $H$ is strongly metrically regular at $\bar{x}$ for $\bar{y}$; that is, the mapping $y \mapsto H^{-1}(y) \cap \mathbb{B}_{a}(\bar{x})$ is single-valued and Lipschitz continuous on $\mathbb{B}_{b}(\bar{y})$ with a Lipschitz constant $\kappa$, then for $\mu, \kappa^{\prime}, \alpha$ and $\beta$ as above and any function $h$ satisfying (7) and (8), the mapping $y \mapsto(h+H)^{-1}(y) \cap \mathbb{B}_{\alpha}(\bar{x})$ is a Lipschitz continuous function on $\mathbb{B}_{\beta}(\bar{y})$ with a Lipschitz constant $\kappa^{\prime} /(1-\kappa \mu)$. 
Before proving the theorem we will make some comments. If we assume $h(\bar{x})=0$ then Theorem 2.2 simply says that if $H$ is (strongly) metrically regular at $\bar{x}$ for $\bar{y}$ and $h$ has a sufficiently small Lipschitz constant, then the perturbed $h+H$ is (strongly) metrically regular at $\bar{x}$ for $\bar{y}$; indeed in this case the claim involving (9) means that $(h+H)^{-1}$ has the Aubin property at $\bar{y}$ for $\bar{x}$ which is equivalent to metric regularity of $h+H$ at $\bar{x}$ for $\bar{y}$, and then we obtain the (extended) LyustenikGraves theorem as stated in [11, Theorem 5E.1]. For the strong regularity part we get a version of Robinson's theorem, see [11, Theorem 5F.1]. However, if $h(\bar{x}) \neq 0$ then $(\bar{x}, \bar{y})$ may be not in the graph of $h+H$ and then we cannot claim that $h+H$ is (strongly) metrically regular at $\bar{x}$ for $\bar{y}$. Of course, this could be handled by choosing a new function $\tilde{h}$ with $\tilde{h}(x)=h(x)-h(\bar{x})$ and then apply [11, Theorem 5F.1] but the latter does not specify how the constants (e.g. the radii of the balls involved) depend on the data of the problem, which is the crux of the matter in obtaining estimates in the following section. Clearly, the result in Theorem 2.2 is parallel to [11, Theorem 5F.1] and can be recovered from the latter; still, but we feel that giving a complete proof would be beneficial for the reader.

Proof. Choose $\mu$ and $\kappa^{\prime}$ as required and then $\alpha$ and $\beta$ to satisfy (6). For any $x \in \mathbb{B}_{\alpha}(\bar{x})$ and $y \in \mathbb{B}_{\beta}(\bar{y})$, using the shift-invariance of the metric in $Y,(7)$, (8) and the triangle inequality, we obtain

$$
\rho(-h(x)+y, \bar{y}) \leq \rho(0, h(\bar{x}))+\rho(h(\bar{x}), h(x))+\rho(y, \bar{y}) \leq \beta+\mu \rho(x, \bar{x})+\beta \leq 2 \beta+\mu \alpha \leq b,
$$

where the last inequality follows from the second inequality in (6). Fix $y^{\prime} \in \mathbb{B}_{\beta}(\bar{y})$ and consider the mapping

$$
\Phi_{y^{\prime}}: x \mapsto H^{-1}\left(-h(x)+y^{\prime}\right) \quad \text { for } \quad x \in \mathbb{B}_{\alpha}(\bar{x}) .
$$

Clearly, gph $\Phi_{y^{\prime}}$ is closed. Let $y \in \mathbb{B}_{\beta}(\bar{y}), y \neq y^{\prime}$ and let $x \in(h+H)^{-1}(y) \cap \mathbb{B}_{\alpha}(\bar{x})$. We will apply Theorem 2.1 with the complete metric space $X$ identified with the closed ball $\mathbb{B}_{\alpha}(\bar{x})$ to show that there is a fixed point $x^{\prime} \in \Phi_{y}\left(x^{\prime}\right)$ in the closed ball centered at $x$ with radius

$$
\varepsilon:=\frac{\kappa^{\prime} \rho\left(y, y^{\prime}\right)}{1-\kappa \mu} .
$$

Then, from the third inequality in (6), we obtain

$$
\varepsilon \leq \frac{\kappa^{\prime}(2 \beta)}{1-\kappa \mu} \leq \alpha .
$$

Hence, from the first inequality in (6) we get $\mathbb{B}_{\varepsilon}(x) \subset \mathbb{B}_{a}(\bar{x})$. Since $y \in h(x)+H(x)$ and $(x, y)$ satisfies (10), from the assumed metric regularity of $H$ we get

$$
\begin{aligned}
d\left(x, \Phi_{y^{\prime}}(x)\right) & =d\left(x, H^{-1}\left(-h(x)+y^{\prime}\right)\right) \leq \kappa d\left(-h(x)+y^{\prime}, H(x)\right) \\
& =\kappa d\left(y^{\prime}, h(x)+H(x)\right) \leq \kappa \rho\left(y, y^{\prime}\right) \\
& <\kappa^{\prime} \rho\left(y, y^{\prime}\right)=\varepsilon(1-\kappa \mu) .
\end{aligned}
$$

For any $u, v \in \mathbb{B}_{\varepsilon}(x)$, using (8), we have

$$
e\left(\Phi_{y^{\prime}}(u) \cap \mathbb{B}_{\varepsilon}(x), \Phi_{y^{\prime}}(v)\right) \leq e\left(H^{-1}\left(-h(u)+y^{\prime}\right) \cap \mathbb{B}_{a}(\bar{x}), H^{-1}\left(-h(v)+y^{\prime}\right)\right)
$$




$$
\leq \kappa \rho(h(u), h(v)) \leq \kappa \mu \rho(u, v)
$$

Applying Theorem 2.1 to the mapping $\Phi_{y^{\prime}}$, with $\bar{x}$ identified with $x$ and constants $a=\varepsilon$ and $\theta=\kappa \mu$, we obtain the existence of a fixed point $x^{\prime} \in \Phi_{y^{\prime}}\left(x^{\prime}\right)=H^{-1}\left(-h\left(x^{\prime}\right)+y^{\prime}\right)$, which is equivalent to $x^{\prime} \in(h+H)^{-1}\left(y^{\prime}\right)$, within distance $\varepsilon$ given by (11) from $x$.

For the second part of the theorem, suppose that $y \mapsto s(y):=H^{-1}(y) \cap \mathbb{B}_{a}(\bar{x})$ is a Lipschitz continuous function on $\mathbb{B}_{b}(\bar{y})$ with a Lipschitz constant $\kappa$. Choose $\mu, \kappa^{\prime}, \alpha$ and $\beta$ as in the statement and let $h$ satisfy $(7)$ and (8). For any $y \in \mathbb{B}_{\beta}(\bar{y})$, since $\bar{x} \in(h+H)^{-1}(\bar{y}+h(\bar{x})) \cap \mathbb{B}_{\alpha}(\bar{x})$, from $(9)$ we obtain that there exists $x \in(h+H)^{-1}(y)$ such that

$$
\rho(x, \bar{x}) \leq \frac{\kappa^{\prime}}{1-\kappa \mu} \rho(y, \bar{y}+h(\bar{x})) .
$$

Since $\rho(y, \bar{y}+h(\bar{x})) \leq 2 \beta$, by $(6)$ we get $\rho(x, \bar{x}) \leq \alpha$, that is, $(h+H)^{-1}(y) \cap \mathbb{B}_{\alpha}(\bar{x}) \neq \emptyset$. Hence the domain of the mapping $(h+H)^{-1} \cap \mathbb{B}_{\alpha}(\bar{x})$ contains $\mathbb{B}_{\beta}(\bar{y})$.

If $x \in(h+H)^{-1}(y) \cap \mathbb{B}_{\alpha}(\bar{x})$, then $x \in H^{-1}(y-h(x)) \cap \mathbb{B}_{\alpha}(\bar{x}) \subset H^{-1}(y-h(x)) \cap \mathbb{B}_{a}(\bar{x})=$ $s(y-h(x))$ since $y-h(x) \in \mathbb{B}_{b}(\bar{y})$ according to $(10)$. Hence,

$$
H^{-1}(y-h(x)) \cap \mathbb{B}_{\alpha}(\bar{x})=s(y-h(x))=x .
$$

Assume that there exist $y \in \mathbb{B}_{\beta}(\bar{y})$ and $x, x^{\prime} \in(h+H)^{-1}(y) \cap \mathbb{B}_{\alpha}(\bar{x})$ such that $x \neq x^{\prime}$. From $(10)$ we have that both $y-h(x)$ and $y-h\left(x^{\prime}\right)$ are in $\mathbb{B}_{b}(\bar{y})$. Then from (12) we get

$$
\begin{aligned}
\rho\left(x, x^{\prime}\right) & =\rho\left(s\left(-h\left(x^{\prime}\right)+y\right), s(-h(x)+y)\right) \\
& \leq \kappa \rho\left(-h\left(x^{\prime}\right)+y,-h(x)+y\right)=\kappa \rho\left(h\left(x^{\prime}\right), h(x)\right) \\
& \leq \kappa \mu \rho\left(x^{\prime}, x\right)<\rho\left(x^{\prime}, x\right)
\end{aligned}
$$

which is a contradiction. Hence, the mapping $y \mapsto g(y):=(h+H)^{-1}(y) \cap \mathbb{B}_{\alpha}(\bar{x})$ is singlevalued, that is, a function, defined on $\mathbb{B}_{\beta}(\bar{y})$. Let $y, y^{\prime} \in \mathbb{B}_{\beta}(\bar{y})$. Utilizing the equality $g(y)=$ $s(-h(g(y))+y)$, see $(12)$, we have

$$
\begin{aligned}
\rho\left(g(y), g\left(y^{\prime}\right)\right) & \left.=\rho\left(s(-h(g(y))+y), s\left(-h\left(g\left(y^{\prime}\right)\right)+y^{\prime}\right)\right)\right) \\
& \leq \kappa \rho\left(h(g(y)), h\left(g\left(y^{\prime}\right)\right)\right)+\kappa \rho\left(y, y^{\prime}\right) \\
& \leq \kappa \mu \rho\left(g(y), g\left(y^{\prime}\right)\right)+\kappa \rho\left(y, y^{\prime}\right) .
\end{aligned}
$$

Thus,

$$
\rho\left(g(y), g\left(y^{\prime}\right)\right) \leq \frac{\kappa^{\prime}}{1-\kappa \mu} \rho\left(y, y^{\prime}\right)
$$

that is, $g$ is Lipschitz continuous with Lipschitz constant $\kappa^{\prime} /(1-\kappa \mu)$. The proof is complete.

\section{Convergence under metric regularity}

In this section we show that under certain conditions, the quasi-Newton iteration (4) is locally q-linearly convergent. We note that in (4) the update $B_{k+1}$ is usually defined in terms of $B_{k}, x_{k}$, and $x_{k+1}$. This is the case in the Broyden update considered in the next section, for which we show that the sufficient conditions for q-linear convergence are satisfied and we are able to get q-superlinear convergence under stronger conditions. 
Theorem 3.1. Suppose that $f+F$ is metrically regular at $\bar{x}$ for 0 with constant $\lambda$. Then, in particular, $\bar{x}$ is a solution of (1). Consider the quasi-Newton method (4) and assume that

$$
\left\|B_{0}-\operatorname{Df}(\bar{x})\right\|<1 /(2 \lambda) .
$$

Furthermore, assume that there exist a constant $c>0$ and a neighborhood $U$ of $\bar{x}$ such that, for $k=0,1, \ldots$, and for any $B_{k}$ and any $x_{k}, x_{k+1} \in U, x_{k} \neq x_{k+1}$, all satisfying (4), the operator $B_{k+1}$ is chosen in such a way that

$$
\left\|B_{k+1}-D f(\bar{x})\right\| \leq\left\|B_{k}-D f(\bar{x})\right\|+c\left(\left\|x_{k}-\bar{x}\right\|+\left\|x_{k+1}-\bar{x}\right\|\right) .
$$

Then there exists a neighborhood $O$ of $\bar{x}$ such that for any $x_{0} \in O$ there exists a sequence $\left\{x_{k}\right\}$ starting at $x_{0}$ and generated by (4) which stays in $O$ and is q-linearly convergent to a solution to (1); moreover, if $x_{k+1} \neq x_{k}$ for all $k$ then $\left\{x_{k}\right\}$ converges to $\bar{x}$. If in addition $f+F$ is strongly metrically regular at $\bar{x}$ for 0 then there is a unique in $O$ sequence $\left\{x_{k}\right\}$ starting at $x_{0}$ and generated by (4), and hence it converges q-linearly to $\bar{x}$.

Proof. Choose $\kappa>\lambda$ such that

$$
\delta:=\left\|B_{0}-D f(\bar{x})\right\|<1 /(2 \kappa)
$$

Let $\kappa^{\prime}>\kappa$ be such that

$$
\frac{\kappa^{\prime} \delta}{1-\kappa \delta}<1
$$

and then fix $\gamma>0$ to satisfy

$$
\frac{\kappa^{\prime} \delta}{1-\kappa \delta}<\gamma<1
$$

Choose $\varepsilon>0$ such that

$$
\frac{\kappa^{\prime}}{1-\kappa(\delta+\varepsilon)}(\varepsilon+\delta)<\gamma
$$

Let

$$
H(x)=f(\bar{x})+D f(\bar{x})(x-\bar{x})+F(x),
$$

for $x \in X$. From Theorem 2.2 applied to $f+F$ and $h(\cdot)=f(\bar{x})+D f(\bar{x})(\cdot-\bar{x})-f(\cdot)$ (or simply by the standard Lyusternik-Graves theorem, see e.g. [11, Theorem 5E.1]), since $h(\bar{x})=0, \kappa>\lambda$, and $(f+F)+h=H$, then (9) implies metric regularity of $H$ at $\bar{x}$ for 0 with constant $\kappa$. Thus, it follows the existence of some positive constants $a$ and $b$ such that

$$
d\left(x, H^{-1}(y)\right) \leq \kappa d(y, H(x)) \quad \text { for all } \quad x \in \mathbb{B}_{a}(\bar{x}), y \in \mathbb{B}_{b}(0) .
$$

Using (16), make $a$ smaller if necessary so that $\mathbb{B}_{a}(\bar{x}) \subset U$,

$$
\begin{gathered}
\|f(u)-f(v)-D f(\bar{x})(u-v)\| \leq \varepsilon\|u-v\| \quad \text { for all } u, v \in \mathbb{B}_{a}(\bar{x}), \\
\kappa\left(\delta+\frac{c a}{1-\gamma}\right)<1
\end{gathered}
$$

and

$$
\frac{\kappa^{\prime}}{1-\kappa\left(\delta+\frac{c a}{1-\gamma}\right)}\left(\varepsilon+\delta+\frac{c a}{1-\gamma}\right)<\gamma
$$


where $c$ is from (14). Set

$$
\mu:=\delta+\frac{c a}{1-\gamma}
$$

and choose positive $\alpha$ and $\beta$ to satisfy the inequalities (6) in Theorem 2.2. Choose $\tau \in(0, \alpha)$ such that

$$
\left(\varepsilon+\delta+\frac{c a}{1-\gamma}\right) \tau \leq \beta
$$

Denote $O:=\mathbb{B}_{\tau}(\bar{x})$ and choose any $x_{0} \in O \backslash\{\bar{x}\}$. Consider the function

$$
h_{0}(x)=f\left(x_{0}\right)+B_{0}\left(x-x_{0}\right)-f(\bar{x})-D f(\bar{x})(x-\bar{x}) .
$$

Then we have

$$
\bar{y}_{0} \in h_{0}(\bar{x})+H(\bar{x}) \quad \text { where } \quad \bar{y}_{0}:=f\left(x_{0}\right)-f(\bar{x})+B_{0}\left(\bar{x}-x_{0}\right) .
$$

Further,

$$
\begin{aligned}
\left\|h_{0}(\bar{x})\right\| & =\left\|f\left(x_{0}\right)+B_{0}\left(\bar{x}-x_{0}\right)-f(\bar{x})\right\| \\
& \leq\left\|f\left(x_{0}\right)-f(\bar{x})-D f(\bar{x})\left(x_{0}-\bar{x}\right)\right\|+\left\|\left(B_{0}-D f(\bar{x})\right)\left(\bar{x}-x_{0}\right)\right\| \\
& \leq(\varepsilon+\delta) \tau \leq \beta
\end{aligned}
$$

where we use (20). For any $x, x^{\prime} \in \mathbb{B}_{\alpha}(\bar{x})$ from (15) we have

$$
\left\|h_{0}(x)-h_{0}\left(x^{\prime}\right)\right\|=\left\|\left(B_{0}-D f(\bar{x})\right)\left(x-x^{\prime}\right)\right\| \leq \delta\left\|x-x^{\prime}\right\| \leq \mu\left\|x-x^{\prime}\right\| .
$$

Also, observe that $\bar{y}_{0}=h_{0}(\bar{x})$, hence from (22) we get $\bar{y}_{0} \in \mathbb{B}_{\beta}(0)$. Finally, from (21) we have $\bar{x} \in\left(h_{0}+H\right)^{-1}\left(\bar{y}_{0}\right)$. We are now ready to apply Theorem 2.2 with $H$ defined in $(17), h=h_{0}, \kappa$, $\mu, \kappa^{\prime}, a, b, \alpha, \beta$ having the values defined above, to obtain that there exists $x_{1} \in\left(h_{0}+H\right)^{-1}(0)$, that is, $x_{1}$ satisfies (4) for $k=0$, and also

$$
\left\|x_{1}-\bar{x}\right\| \leq \frac{\kappa^{\prime}}{1-\kappa \mu}\left\|\bar{y}_{0}\right\| \leq \frac{\kappa^{\prime}}{1-\kappa \mu}(\varepsilon+\delta)\left\|x_{0}-\bar{x}\right\| \leq \gamma\left\|x_{0}-\bar{x}\right\|,
$$

where we use the estimates (19) and (22). Since $\gamma<1$, this yields $x_{1} \in O=\mathbb{B}_{\tau}(\bar{x})$.

If $x_{1}=\bar{x}$, by taking $x_{k}=\bar{x}$ for all $k>1$ we are done. If $x_{1}=x_{0}$, by taking $x_{k}=x_{0}$ for all $k>1$ we are also done. Otherwise, we use induction which is somewhat parallel to the first step of the proof with some important differences. Suppose that there exist an integer $n>1$ and points $x_{1}, \ldots, x_{n}$ with $x_{k} \in \mathbb{B}_{\tau}(\bar{x}), x_{k-1} \neq x_{k} \neq \bar{x}$ and

$$
\left\|x_{k}-\bar{x}\right\| \leq \gamma\left\|x_{k-1}-\bar{x}\right\| \quad \text { for } \quad k=1, \ldots, n .
$$

From condition (14) and taking into account that $\tau \leq \alpha \leq a / 2$ we get

$$
\begin{aligned}
\left\|D f(\bar{x})-B_{n}\right\| & \leq\left\|D f(\bar{x})-B_{0}\right\|+c \sum_{k=1}^{n}\left(\left\|x_{k}-\bar{x}\right\|+\left\|\bar{x}-x_{k-1}\right\|\right) \\
& \leq \delta+c \sum_{k=1}^{n}\left(\left\|x_{k}-\bar{x}\right\|+\left\|\bar{x}-x_{k-1}\right\|\right)
\end{aligned}
$$




$$
\leq \delta+2 c \sum_{k=0}^{n}\left\|x_{k}-\bar{x}\right\| \leq \delta+2 c \sum_{k=0}^{\infty} \gamma^{k}\left\|x_{0}-\bar{x}\right\| \leq \delta+\frac{c a}{1-\gamma}
$$

Define

$$
h_{n}(x)=f\left(x_{n}\right)+B_{n}\left(x-x_{n}\right)-f(\bar{x})-D f(\bar{x})(x-\bar{x})
$$

and

$$
\bar{y}_{n}:=f\left(x_{n}\right)-f(\bar{x})+B_{n}\left(\bar{x}-x_{n}\right) .
$$

Then, using (24), we obtain

$$
\begin{aligned}
\left\|\bar{y}_{n}\right\| & =\left\|f\left(x_{n}\right)-f(\bar{x})-D f(\bar{x})\left(x_{n}-\bar{x}\right)\right\|+\left\|\left(B_{n}-D f(\bar{x})\right)\left(\bar{x}-x_{n}\right)\right\| \\
& \leq\left(\varepsilon+\delta+\frac{c a}{1-\gamma}\right)\left\|x_{n}-\bar{x}\right\| \leq\left(\varepsilon+\delta+\frac{c a}{1-\gamma}\right)\left\|x_{0}-\bar{x}\right\| .
\end{aligned}
$$

Hence, by (20),

$$
\left\|\bar{y}_{n}\right\| \leq\left(\varepsilon+\delta+\frac{c a}{1-\gamma}\right) \tau \leq \beta
$$

Since $h_{n}(\bar{x})=\bar{y}_{n}$, we get $\left\|h_{n}(\bar{x})\right\| \leq \beta$. Also, for any $x, x^{\prime} \in \mathbb{B}_{\alpha}(\bar{x})$ we obtain

$$
\left\|h_{n}(x)-h_{n}\left(x^{\prime}\right)\right\|=\left\|\left(B_{n}-D f(\bar{x})\right)\left(x-x^{\prime}\right)\right\| \leq\left(\delta+\frac{c a}{1-\gamma}\right)\left\|x-x^{\prime}\right\|=\mu\left\|x-x^{\prime}\right\| .
$$

The assumptions of Theorem 2.2 are then satisfied, hence, taking into account that $\bar{x} \in\left(h_{n}+\right.$ $H)^{-1}\left(\bar{y}_{n}\right)$ we conclude that there exists $x_{n+1} \in\left(h_{n}+H\right)^{-1}(0)$, that is, satisfying (4) for $k=n$, such that

$$
\left\|x_{n+1}-\bar{x}\right\| \leq \frac{\kappa^{\prime}}{1-\kappa \mu}\left\|\bar{y}_{n}\right\| .
$$

Then, utilizing (19) and (25) we obtain

$$
\left\|x_{n+1}-\bar{x}\right\| \leq \frac{\kappa^{\prime}}{1-\kappa \mu}\left(\varepsilon+\delta+\frac{c a}{1-\gamma}\right)\left\|x_{n}-\bar{x}\right\| \leq \gamma\left\|x_{n}-\bar{x}\right\| .
$$

Hence, $x_{n+1} \in \mathbb{B}_{\tau}(\bar{x})$ and the induction step is complete. Since $\gamma<1$, if $x_{n} \neq x_{n+1}$ for all $n$, the sequence $x_{k}$ converges to $\bar{x}$ q-linearly. Otherwise the sequence converges in a finite number of steps to a solution to the equation (1).

For the final statement, when $f+F$ is strongly metrically regular, then, according to the second part of Theorem 2.2, the point $x_{n+1} \in\left(h_{n}+H\right)^{-1}(0)$ is unique in $O$. Furthermore, $(f+F)^{-1} \cap O=\{\bar{x}\}$ and hence the sequence must converge to $\bar{x}$. The proof is complete.

\section{Convergence of the Broyden update}

In this section $X$ and $Y$ are real Hilbert spaces with scalar products denoted by $\langle\cdot, \cdot\rangle$. We consider the following well-known Broyden update:

$$
B_{k+1}:=B_{k}+\frac{\left(y_{k}-B_{k} s_{k}\right)\left\langle s_{k}, \cdot\right\rangle}{\left\|s_{k}\right\|^{2}}
$$


where $y_{k}:=f\left(x_{k+1}\right)-f\left(x_{k}\right)$ and $s_{k}:=x_{k+1}-x_{k}$. Usually, $B_{0}$ is taken as $D f\left(x_{0}\right)$.

There are quite a few papers dealing with quasi-Newton methods for solving the nonlinear equations in the infinite-dimensional setting, and some of them use the Broyden update, see e.g. [3], [13], [15], [18], [22], [23], [25]. In some of these papers, such as [15] and [18], $Y$ is a Banach space; in [15] $X$ is also a Banach space having a continuous inner product, and with (27) being defined on the completion of $X$ in the norm induced by this inner product.

We apply Theorem 3.1 to the Broyden update (27) showing that it satisfies condition (14) and hence is q-linearly convergent, locally.

We start with an elementary lemma.

Lemma 4.1. Let $A \in \mathcal{L}(X, Y)$. If $x \in X \backslash\{0\}$, then

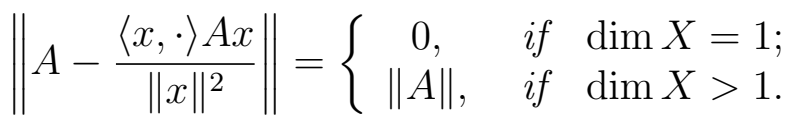

Proof. Let $z \in \operatorname{span}(x)=\{\lambda x \mid \lambda \in \mathbb{R}\}$. Then there is some $\lambda_{0} \in \mathbb{R}$ such that $z=\lambda_{0} x$, from where,

$$
\left\|A z-\frac{\langle x, z\rangle A x}{\|x\|^{2}}\right\|=\left\|\lambda_{0} A x-\frac{\lambda_{0}\langle x, x\rangle A x}{\|x\|^{2}}\right\|=0 .
$$

If $\operatorname{dim} X=1$, then $X=\operatorname{span}(x)$, and from the above equality we obtain (28). Otherwise, assume that $\operatorname{dim} X>1$. For any $z \in \mathbb{B}_{X}$, one has

$$
\left\|z-\frac{\langle x, z\rangle x}{\|x\|^{2}}\right\|^{2}=\|z\|^{2}-\frac{\langle x, z\rangle^{2}}{\|x\|^{2}} \leq 1 .
$$

Hence,

$$
\left\|A-\frac{\langle x, \cdot\rangle A x}{\|x\|^{2}}\right\|=\sup _{w \in \mathbb{B}_{X}}\left\|A\left(w-\frac{\langle x, w\rangle x}{\|x\|^{2}}\right)\right\| \leq\|A\| \sup _{w \in \mathbb{B}_{X}}\left\|w-\frac{\langle x, w\rangle x}{\|x\|^{2}}\right\| \leq\|A\| .
$$

For any $z \in\{x\}^{\perp}=\{w \in X \mid\langle w, x\rangle=0\} \neq \emptyset$, one has

$$
\left\|A z-\frac{\langle x, z\rangle A x}{\|x\|^{2}}\right\|=\|A z\|,
$$

and therefore (28) follows.

The following result is a generalization to Hilbert spaces of a statement included in the first part of the proof of [24, Th. 5.4.13].

Proposition 4.2. Suppose that the Fréchet derivative mapping Df is Lipschitz continuous with constant $L$ in a convex neighborhood $U$ of a point $\bar{x}$. Given $B_{k} \in \mathcal{L}(X, Y)$ and $x_{k}, x_{k+1} \in U$, with $x_{k+1} \neq x_{k}$, if $B_{k+1}$ is defined as in (27), then

$$
\left\|B_{k+1}-D f(\bar{x})\right\| \leq\left\|B_{k}-D f(\bar{x})\right\|+\frac{L}{2}\left(\left\|x_{k+1}-\bar{x}\right\|+\left\|\bar{x}-x_{k}\right\|\right) .
$$


Proof. By assumption,

$$
\|D f(u)-D f(v)\| \leq L\|u-v\| \quad \text { for all } u, v \in U .
$$

Let $x_{k+1}, x_{k} \in U, x_{k} \neq x_{k+1}$ and let $B_{k+1}$ be defined as in (27). Then

$$
\begin{aligned}
B_{k+1}-D f(\bar{x}) & =B_{k}-D f(\bar{x})+\frac{\left(y_{k}-B_{k} s_{k}\right)\left\langle s_{k}, \cdot\right\rangle}{\left\|s_{k}\right\|^{2}} \\
& =B_{k}-D f(\bar{x})-\frac{\left(B_{k}-D f(\bar{x})\right) s_{k}\left\langle s_{k}, \cdot\right\rangle}{\left\|s_{k}\right\|^{2}}+\frac{\left(y_{k}-D f(\bar{x}) s_{k}\right)\left\langle s_{k}, \cdot\right\rangle}{\left\|s_{k}\right\|^{2}} .
\end{aligned}
$$

Thus,

$$
\left\|B_{k+1}-D f(\bar{x})\right\| \leq\left\|\left(B_{k}-D f(\bar{x})\right)-\frac{\left(B_{k}-D f(\bar{x})\right) s_{k}\left\langle s_{k}, \cdot\right\rangle}{\left\|s_{k}\right\|^{2}}\right\|+\frac{\left\|y_{k}-D f(\bar{x}) s_{k}\right\|}{\left\|s_{k}\right\|} .
$$

By Lemma 4.1,

$$
\left\|\left(B_{k}-D f(\bar{x})\right)-\frac{\left(B_{k}-D f(\bar{x})\right) s_{k}\left\langle s_{k}, \cdot\right\rangle}{\left\|s_{k}\right\|^{2}}\right\| \leq\left\|B_{k}-D f(\bar{x})\right\| .
$$

Utilizing the mean value theorem we obtain

$$
\begin{aligned}
\left\|y_{k}-D f(\bar{x}) s_{k}\right\| & =\left\|f\left(x_{k+1}\right)-f\left(x_{k}\right)-D f(\bar{x}) s_{k}\right\| \\
& =\left\|\int_{0}^{1}\left[D f\left(x_{k}+t\left(x_{k+1}-x_{k}\right)\right)\left(x_{k+1}-x_{k}\right)-D f(\bar{x}) s_{k}\right] d t\right\| \\
& \leq\left\|s_{k}\right\| \int_{0}^{1}\left\|D f\left(x_{k}+t\left(x_{k+1}-x_{k}\right)\right)-D f(\bar{x})\right\| d t \\
& \leq L\left\|s_{k}\right\| \int_{0}^{1}\left((1-t)\left\|x_{k}-\bar{x}\right\|+t\left\|x_{k+1}-\bar{x}\right\|\right) d t \\
& =\frac{L}{2}\left\|s_{k}\right\|\left(\left\|x_{k+1}-\bar{x}\right\|+\left\|x_{k}-\bar{x}\right\|\right) .
\end{aligned}
$$

This yields (29).

Thus, applying Theorem 3.1 we obtain the following result:

Theorem 4.3. Consider the generalized equation (1) in the setting of Hilbert spaces $X$ and $Y$ with a solution $\bar{x}$ and suppose that the derivative mapping $D f$ is Lipschitz continuous around $\bar{x}$. Also, suppose that $f+F$ is metrically regular at $\bar{x}$ for 0 with constant $\lambda$. Consider the quasi-Newton method (4) applied to (1) with the Broyden update (27) and with $B_{0}$ satisfying (13). Then there exists a neighborhood $O$ of $\bar{x}$ such that for any $x_{0} \in O$ there exists a sequence $\left\{x_{k}\right\}$ starting from $x_{0}$ and generated by (4) which stays in $O$ and is q-linearly convergent; moreover, if $x_{k+1} \neq x_{k}$ then the sequence is q-linearly convergent to $\bar{x}$. If in addition $f+F$ is strongly metrically regular at $\bar{x}$ for 0 then there is a unique in $O$ sequence $\left\{x_{k}\right\}$ starting from $x_{0}$ and generated by (4), and hence it converges q-linearly to $\bar{x}$. 
We devote the remainder of this section to the q-superlinear convergence of the Broyden update, that is, the mode of convergence satisfying

$$
\lim _{k \rightarrow \infty} \frac{\left\|e_{k+1}\right\|}{\left\|e_{k}\right\|}=0
$$

where $e_{k}=x_{k}-\bar{x}$.

Recall that the Hilbert-Schmidt norm of an operator $A \in \mathcal{L}(X, Y)$ is defined as

$$
\|A\|_{H S}=\sqrt{\sum_{i \in I}\left\|A e_{i}\right\|^{2}}
$$

where $\left\{e_{i}, i \in I\right\}$ is an orthonormal basis of $X$. Denote $\mathcal{H}(X, Y)=\left\{A \in \mathcal{L}(X, Y) \mid\|A\|_{H S}<+\infty\right\}$. Endowed with the inner product

$$
\langle A, B\rangle_{H S}=\sum_{i \in I}\left\langle A e_{i}, B e_{i}\right\rangle
$$

$\mathcal{H}(X, Y)$ becomes a Hilbert space, see [20]. In Euclidean spaces this norm coincides with the Frobenius norm.

We start with a lemma which echoes Lemma 4.1.

Lemma 4.4. Let $A \in \mathcal{H}(X, Y)$. If $0 \neq x \in X$, then

$$
\left\|A-\frac{\langle x, \cdot\rangle A x}{\|x\|^{2}}\right\|_{H S}^{2}=\|A\|_{H S}^{2}-\frac{\|A x\|^{2}}{\|x\|^{2}} .
$$

Proof. Note that

$$
\left\|A-\frac{\langle x, \cdot\rangle A x}{\|x\|^{2}}\right\|_{H S}^{2}=\|A\|_{H S}^{2}+\left\|\frac{\langle x, \cdot\rangle A x}{\|x\|^{2}}\right\|_{H S}^{2}-2\left\langle A, \frac{\langle x, \cdot\rangle A x}{\|x\|^{2}}\right\rangle_{H S} .
$$

Further, by the Parseval identity,

$$
\|\langle x, \cdot\rangle A x\|_{H S}^{2}=\sum_{i \in I}\left\|\left\langle x, e_{i}\right\rangle A x\right\|^{2}=\|A x\|^{2} \sum_{i \in I}\left\langle x, e_{i}\right\rangle^{2}=\|A x\|^{2}\|x\|^{2},
$$

and

$$
\langle A,\langle x, \cdot\rangle A x\rangle_{H S}=\sum_{i \in I}\left\langle A e_{i},\left\langle x, e_{i}\right\rangle A x\right\rangle=\sum_{i \in I}\left\langle A\left\langle x, e_{i}\right\rangle e_{i}, A x\right\rangle=\|A x\|^{2},
$$

where to get the last equality we apply Remark 1.2.1(c) in [20]. This yields (31).

Lemma 4.4 implies that the Proposition 4.2 is valid also for the Hilbert-Schmidt norm.

Proposition 4.5. Suppose that the derivative mapping $D f$ is Lipschitz continuous with respect to the Hilbert-Schmidt norm with constant $L$ in a convex neighborhood $U$ of a point $\bar{x}$. Given $B_{k} \in \mathcal{H}(X, Y)$ and $x_{k}, x_{k+1} \in U$, with $x_{k+1} \neq x_{k}$, if $B_{k+1}$ is defined as in (27), then

$$
\left\|B_{k+1}-D f(\bar{x})\right\|_{H S} \leq\left\|B_{k}-D f(\bar{x})\right\|_{H S}+\frac{L}{2}\left(\left\|x_{k+1}-\bar{x}\right\|+\left\|\bar{x}-x_{k}\right\|\right) .
$$


Proof. This can be obtained by applying the same argument as in Proposition 4.2 but using Lemma 4.4 instead of Lemma 4.1.

Corollary 4.6. If $B_{0}-D f(\bar{x})$ is a Hilbert-Schmidt operator and $D f$ is Lipschitz with respect to the Hilbert-Schmidt norm, then $B_{k}-D f(\bar{x})$ is a Hilbert-Schmidt operator, for all $k \in \mathbb{N}$.

Proof. This follows from (32).

The following proposition is the main stepping stone to obtain q-superlinear convergence. It links to analysis presented so far with a central result in the theory of quasi-Newton methods the Dennis-Moré theorem. This theorem, first published in [5], gives a characterization for the q-superlinear convergence of a quasi-Newton method applied to a smooth equation $f(x)=0$ with a zero at $\bar{x}$ at which the derivative mapping $D f(\bar{x})$ is invertible. Namely, if a quasi-Newton method generates a sequence $\left\{x_{k}\right\}$ which stays near $\bar{x}$ and $x_{k+1} \neq x_{k}$ for all $k$, then $\left\{x_{k}\right\}$ is convergent q-superlinearly if and only if it is convergent and, in addition,

$$
\lim _{k \rightarrow \infty} \frac{\left\|E_{k} s_{k}\right\|}{\left\|s_{k}\right\|}=0,
$$

where $E_{k}:=B_{k}-D f(\bar{x})$.

It is known that the Broyden update (27) applied to a smooth equation in finite dimensions with a nonsingular Jacobian at the reference solution $\bar{x}$ satisfies condition (33), hence in that case the Broyden update (27) converges q-superlinearly, see [17, Theorem 7.2.4]. We will now show that (33) also holds in the setting of generalized equations currently considered. This will allow us to apply the Dennis-Moré theorem for generalized equations in Banach spaces proved in [9, Theorem 3]. For completeness we state next the sufficiency part of this theorem which is used in further lines.

Theorem 4.7. Consider the generalized equation (1) with a solution $\bar{x}$ and suppose that $f$ is Fréchet differentiable in a neighborhood $U$ of $\bar{x}$ and the derivative mapping $D f$ is continuous at $\bar{x}$. Also, suppose that the mapping $x \mapsto f(\bar{x})+D f(\bar{x})(x-\bar{x})+F(x)$ is strongly metrically subregular at $\bar{x}$ for 0 . If a sequence $\left\{x_{k}\right\}$ generated by (4) is convergent to $\bar{x}$ and satisfies (33), then it is convergent q-superlinearly.

The following proposition gives conditions under which the Broyden update satisfies the DennisMoré condition (33). Observe that this result is new even in finite dimensions.

Proposition 4.8. Consider the generalized equation (1) with a solution $\bar{x}$ and suppose that the derivative mapping $D f$ is Lipschitz continuous around $\bar{x}$ with respect to the Hilbert-Schmidt norm. Consider the quasi-Newton method (4) applied to (1) with the Broyden update (27) such that $B_{0}$ satisfies (13) and $B_{0}-D f(\bar{x})$ is a Hilbert-Schmidt operator. Consider a sequence $\left\{x_{k}\right\}$ generated by (4) which is convergent to $\bar{x}$. Then the Dennis-Moré condition (33) holds.

Proof. We first show that

$$
\lim _{k \rightarrow \infty}\left\|B_{k+1}-B_{k}\right\|_{H S}=0 .
$$


The proof of (34) parallels the analysis in [14], similarly to the proof of [4, Theorem 2.3]. Consider the convex set

$$
\mathcal{C}_{k}:=\left\{A \in \mathcal{L}(X, Y) \mid\|A-D f(\bar{x})\|_{H S}<\infty \text { and } A s_{k}=y_{k}\right\} .
$$

where $s_{k}:=x_{k+1}-x_{k}$ and $y_{k}:=f\left(x_{k+1}\right)-f\left(x_{k}\right)$. Observe that $\mathcal{C}_{k}$ is closed: if $A_{n} \in \mathcal{C}_{k}$ converges to $A \in \mathcal{L}(X, Y)$ (with respect to the Hilbert-Schmidt norm), then

$$
\|A-D f(\bar{x})\|_{H S} \leq\left\|A-A_{n}\right\|_{H S}+\left\|A_{n}-D f(\bar{x})\right\|_{H S}<\infty,
$$

and

$$
\left\|A s_{k}-y_{k}\right\|=\left\|A s_{k}-A_{n} s_{k}\right\| \leq\left\|A-A_{n}\right\|_{H S}\left\|s_{k}\right\|,
$$

where we employ the inequality $\left\|A-B_{k}\right\| \leq\left\|A-B_{k}\right\|_{H S}$ (see e.g. [20, Corollary 16.9]). Taking the limit when $n \rightarrow \infty$, we get $A s_{k}=y_{k}$.

Let $\left\{e_{i}, i \in I\right\}$ be an orthonormal basis of $X$ for an index set $I$. By Corollary 4.6, $B_{k+1} \in \mathcal{C}_{k}$. Moreover, for all $A \in \mathcal{C}_{k}$, one has

$$
\begin{aligned}
\left\|B_{k+1}-B_{k}\right\|_{H S}^{2} & =\left\|\frac{\left(y_{k}-B_{k} s_{k}\right)\left\langle s_{k}, \cdot\right\rangle}{\left\|s_{k}\right\|^{2}}\right\|_{H S}^{2}=\frac{\left\|\left(A-B_{k}\right) s_{k}\left\langle s_{k}, \cdot\right\rangle\right\|_{H S}^{2}}{\left\|s_{k}\right\|^{4}} \\
& =\frac{\sum_{i \in I}\left\|\left(A-B_{k}\right) s_{k}\left\langle s_{k}, e_{i}\right\rangle\right\|^{2}}{\left\|s_{k}\right\|^{4}}=\frac{\sum_{i \in I}\left\langle s_{k}, e_{i}\right\rangle^{2}\left\|\left(A-B_{k}\right) s_{k}\right\|^{2}}{\left\|s_{k}\right\|^{4}} \\
& =\frac{\left\|\left(A-B_{k}\right) s_{k}\right\|^{2}}{\left\|s_{k}\right\|^{2}} \leq\left\|A-B_{k}\right\|_{H S}^{2},
\end{aligned}
$$

where we again use the inequality $\left\|A-B_{k}\right\| \leq\left\|A-B_{k}\right\|_{H S}$. Then Broyden update (27) is the (unique) solution to the minimization problem

$$
\min _{A \in \mathcal{C}_{k}}\left\|A-B_{k}\right\|_{H S} .
$$

Thus, $B_{k+1}$ is the projection of $B_{k}$ onto the closed convex set $\mathcal{C}_{k}$. The projection mapping onto $\mathcal{C}_{k}$, denoted by $P_{\mathcal{C}_{k}}$, is firmly nonexpansive (see e.g. [2, Prop. 4.8]). Therefore, for all $A \in \mathcal{C}_{k}$, one has

$$
\left\|P_{\mathcal{C}_{k}}\left(B_{k}\right)-P_{\mathcal{C}_{k}}(A)\right\|_{H S}^{2}+\left\|\left(I-P_{\mathcal{C}_{k}}\right)\left(B_{k}\right)-\left(I-P_{\mathcal{C}_{k}}\right)(A)\right\|_{H S}^{2} \leq\left\|B_{k}-A\right\|_{H S}^{2},
$$

where $I$ denotes the identity mapping. Hence, for all $A \in \mathcal{C}_{k}$,

$$
\left\|B_{k+1}-A\right\|_{H S}^{2}+\left\|B_{k+1}-B_{k}\right\|_{H S}^{2} \leq\left\|B_{k}-A\right\|_{H S}^{2} .
$$

Define

$$
A_{k}:=\int_{0}^{1} D f\left(x_{k}+t\left(x_{k+1}-x_{k}\right)\right) d t
$$

We have

$$
A_{k} s_{k}=\int_{0}^{1} D f\left(x_{k}+t\left(x_{k+1}-x_{k}\right)\right)\left(x_{k+1}-x_{k}\right) d t=f\left(x_{k+1}\right)-f\left(x_{k}\right)=y_{k} .
$$


Furthermore, since $D f$ is Lipschitz continuous with respect to the Hilbert-Schmidt norm, there is a constant $L \geq 0$ such that, eventually,

$$
\begin{aligned}
\left\|A_{k}-D f(\bar{x})\right\|_{H S} & =\left\|\int_{0}^{1}\left(D f\left(x_{k}+t\left(x_{k+1}-x_{k}\right)\right)-D f(\bar{x})\right) d t\right\|_{H S} \\
& \leq \int_{0}^{1}\left\|D f\left(t x_{k+1}+(1-t) x_{k}\right)-D f(\bar{x})\right\|_{H S} d t \\
& \left.\leq L \int_{0}^{1} \| t\left(x_{k+1}-\bar{x}\right)+(1-t)\left(x_{k}-\bar{x}\right)\right) \| d t \\
& \leq \frac{L}{2}\left(\left\|x_{k+1}-\bar{x}\right\|+\left\|x_{k}-\bar{x}\right\|\right)<\infty .
\end{aligned}
$$

Thus, $A_{k} \in \mathcal{C}_{k}$. Since $x_{k}$ converges to $\bar{x}$, we deduce from (38) that $\left\|A_{k}-\operatorname{Df}(\bar{x})\right\|_{H S}$ converges to zero. Moreover, (32) together with the linear convergence of $x_{k}$ to $\bar{x}$ implies that $\left\|B_{k}-D f(\bar{x})\right\|_{H S}$ is convergent. Indeed, let $0<\gamma<1$ be such that

$$
\left\|x_{k+1}-\bar{x}\right\| \leq \gamma\left\|x_{k}-\bar{x}\right\| \quad \text { for all } k=0,1, \ldots
$$

Then, for all $m>n$, one has by (32)

$$
\begin{aligned}
\left\|B_{m}-D f(\bar{x})\right\|_{H S} & \leq\left\|B_{n}-D f(\bar{x})\right\|_{H S}+\frac{L}{2} \sum_{k=n+1}^{m}\left(\left\|x_{k}-\bar{x}\right\|+\left\|x_{k-1}-\bar{x}\right\|\right) \\
& \leq\left\|B_{n}-D f(\bar{x})\right\|_{H S}+L \sum_{k=n}^{m-1}\left\|x_{k}-\bar{x}\right\| \\
& \leq\left\|B_{n}-D f(\bar{x})\right\|_{H S}+L \sum_{k=n}^{\infty} \gamma^{k}\left\|x_{0}-\bar{x}\right\| \\
& \leq\left\|B_{n}-D f(\bar{x})\right\|_{H S}+\frac{L \gamma^{n}}{1-\gamma}\left\|x_{0}-\bar{x}\right\| .
\end{aligned}
$$

This implies that $\left\|B_{k}-D f(\bar{x})\right\|_{H S}$ is a Cauchy sequence, and thus is convergent. Therefore, since $A_{k}$ defined in (36) converges to $D f(\bar{x})$, we get that $\left\|B_{k}-A_{k}\right\|_{H S}$ and $\left\|B_{k+1}-A_{k}\right\|_{H S}$ converge to the same limit. Furthermore, (35) implies

$$
\left\|B_{k+1}-A_{k}\right\|_{H S}^{2}+\left\|B_{k+1}-B_{k}\right\|_{H S}^{2} \leq\left\|B_{k}-A_{k}\right\|_{H S}^{2}
$$

which in turn yields (34).

We are now ready to prove that (33) is satisfied. Since $\left\|B_{k+1}-B_{k}\right\| \leq\left\|B_{k+1}-B_{k}\right\|_{H S}$, by the triangle inequality we have

$$
\left\|E_{k} s_{k}\right\|=\left\|\left(B_{k}-D f(\bar{x})\right) s_{k}\right\| \leq\left\|\left(B_{k+1}-D f(\bar{x})\right) s_{k}\right\|+\left\|B_{k+1}-B_{k}\right\|_{H S}\left\|s_{k}\right\| .
$$

The next steps mimics the proof of Proposition 4.2. Taking into account that

$$
\left\|\left(B_{k+1}-D f(\bar{x})\right) s_{k}\right\|=\left\|y_{k}-D f(\bar{x}) s_{k}\right\|=\left\|f\left(x_{k+1}\right)-f\left(x_{k}\right)-D f(\bar{x}) s_{k}\right\|
$$


and

$$
f\left(x_{k+1}\right)-f\left(x_{k}\right)=\int_{0}^{1} D f\left(x_{k}+t\left(x_{k+1}-x_{k}\right)\right) s_{k} d t
$$

we get

$$
\begin{aligned}
\left\|\left(B_{k+1}-D f(\bar{x})\right) s_{k}\right\| & \leq\left\|s_{k}\right\| \int_{0}^{1}\left\|D f\left(x_{k}+t\left(x_{k+1}-x_{k}\right)\right)-D f(\bar{x})\right\| d t \\
& \leq\left\|s_{k}\right\| \int_{0}^{1}\left\|D f\left(x_{k}+t\left(x_{k+1}-x_{k}\right)\right)-D f(\bar{x})\right\|_{H S} d t \\
& \leq L\left\|s_{k}\right\| \int_{0}^{1}\left\|t\left(x_{k+1}-\bar{x}\right)+(1-t)\left(x_{k}-\bar{x}\right)\right\| d t \\
& \leq \frac{L\left\|s_{k}\right\|}{2}\left(\left\|x_{k+1}-\bar{x}\right\|+\left\|x_{k}-\bar{x}\right\|\right) .
\end{aligned}
$$

Thus, from (40),

$$
\frac{\left\|E_{k} s_{k}\right\|}{\left\|s_{k}\right\|} \leq \frac{L}{2}\left(\left\|x_{k+1}-\bar{x}\right\|+\left\|x_{k}-\bar{x}\right\|\right)+\left\|B_{k+1}-B_{k}\right\|_{H S} .
$$

Since $\left\|B_{k+1}-B_{k}\right\|_{H S} \rightarrow 0$ by (34) and $x_{k} \rightarrow \bar{x}$, we come to (33).

The following theorem presents the main result of this section.

Theorem 4.9. Consider the generalized equation (1) with a solution $\bar{x}$ and suppose that the derivative mapping $D f$ is Lipschitz continuous around $\bar{x}$ with respect to the Hilbert-Schmidt norm. Consider the quasi-Newton method (4) applied to (1) with the Broyden update (27) such that $B_{0}$ satisfies (13) and $B_{0}-D f(\bar{x})$ is a Hilbert-Schmidt operator.

(i) If $f+F$ is strongly metrically subregular at $\bar{x}$ for 0 , then there exists a neighborhood $O$ of $\bar{x}$ such that any sequence $\left\{x_{k}\right\}$ generated by (4) which converges to $\bar{x}$ is q-superlinearly convergent;

(ii) If $f+F$ is both strongly metrically subregular and metrically regular at $\bar{x}$ for 0 , then there exists a neighborhood $O$ of $\bar{x}$ such that any starting point $x_{0} \in O$ there exists a sequence $\left\{x_{k}\right\}$ generated by (4) which is convergent to $\bar{x}$; moreover, every sequence which is convergent to $\bar{x}$ is q-superlinearly convergent;

(iii) If $f+F$ is strongly metrically regular at $\bar{x}$ for 0 , then there exists a neighborhood $O$ of $\bar{x}$ such that for any $x_{0} \in O$ there exists a unique in $O$ sequence $\left\{x_{k}\right\}$ starting from $x_{0}$ and generated by (4) which is q-superlinearly convergent to $\bar{x}$.

Proof. To prove (i) it is sufficient to combine Proposition 4.8 with Theorem 4.7. Then (ii) follows from (i) and Theorem 4.3. Since strong metric regularity implies strong metric subregularity, in order to to prove (iii) it is sufficient to combine (i) with the last part of Theorem 4.3.

We note that the condition $E_{0}:=B_{0}-D f(\bar{x})$ be a Hilbert-Schmidt operator is used in [23, Theorem 3.5] to prove q-superlinear convergence of the Broyden method for equations. Thus, Theorem 4.7 also extend [23, Theorem 3.5] to generalized equations. 


\section{Two numerical examples}

Our first example is one-dimensional. Let $f: \mathbb{R} \rightarrow \mathbb{R}$ and $F: \mathbb{R} \rightrightarrows \mathbb{R}$ be given by

$$
\begin{aligned}
& f(x):=3 x^{3}-2 x^{2}, \quad \text { for } \quad x \in \mathbb{R} \text {; } \\
& F(x):=\left\{\begin{array}{cc}
\{x,-x\}, & x \geq 0 \\
\emptyset, & x<0 .
\end{array}\right.
\end{aligned}
$$

The graph of $f+F$ is plotted in Figure 5.1. The generalized equation $0 \in f(x)+F(x)$ has two solutions: 0 and 1.

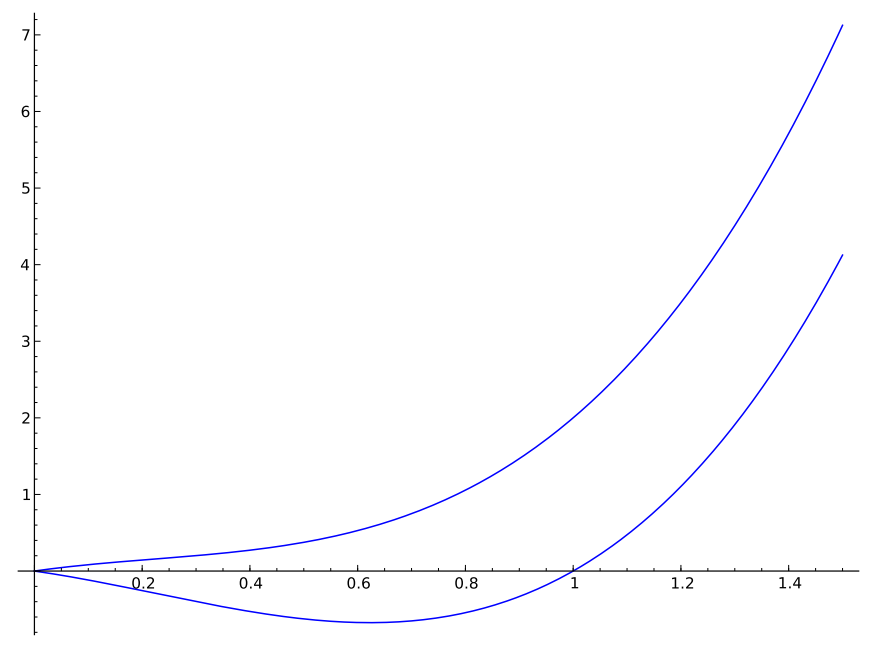

Figure 5.1: The graph of $f+F$ is the union of the graphs of the two curves.

Observe that the mapping $f+F$ is strongly regular at any point of its graph, and particularly at 0 for 0 and at 1 for 0 . Hence the assumptions of Theorem 4.9 are satisfied, and the quasi-Newton method (4) with the Broyden update (27) generates a locally unique q-superlinearly convergent sequence when started within a neighborhood of each of the solutions. The numerical results with $B_{0}:=D f\left(x_{0}\right)$ are shown in Table 5.1 for two staring points: $x_{0}=0.1$ (left) and $x_{0}=0.3$ (right). Note that the obtained convergence is actually q-superlinear in each case.

\begin{tabular}{|c|c|c|c||c|c|c|}
\hline Iteration & $\left\|e_{k}\right\|$ & $\frac{\left\|e_{k+1}\right\|}{\left\|e_{k}\right\|}$ & $\frac{\left\|E_{k} s_{k}\right\|}{\left\|s_{k}\right\|}$ & $\left\|e_{k}\right\|$ & $\frac{\left\|e_{k+1}\right\|}{\left\|e_{k}\right\|}$ & $\frac{\left\|E_{k} s_{k}\right\|}{\left\|s_{k}\right\|}$ \\
\hline 1 & 0.02028986 & 0.20289855 & 0.31000000 & 0.02950820 & 0.09836066 & 0.39000000 \\
\hline 2 & 0.00417421 & 0.20572906 & 0.20325772 & 0.01398735 & 0.47401564 & 0.35984682 \\
\hline 3 & 0.00017129 & 0.04103511 & 0.04738675 & 0.00084106 & 0.06012992 & 0.08255372 \\
\hline 4 & 0.00000143 & 0.00836626 & 0.00863650 & 0.00002369 & 0.02817032 & 0.02903246 \\
\hline 5 & 0.00000000 & 0.00034261 & 0.00034536 & 0.00000004 & 0.00168284 & 0.00172732 \\
\hline 6 & 0.00000000 & 0.00000287 & 0.00000287 & 0.00000000 & 0.00004739 & 0.00004746 \\
\hline
\end{tabular}

Table 5.1. Numerical results for the first example with $x_{0}=0.1$ (left) and $x_{0}=0.3$ (right). 
In the paper [12] the following model of economic equilibrium was introduced. Consider a group of $r$ agents, each of which starts with a vector $x_{i}^{0} \in \mathbb{R}^{n}$ of goods and trades them for another goods vectors $x_{i} \in \mathbb{R}^{n}$. Each good has a price to be determined by the market and the price vector is $p \in \mathbb{R}_{+}^{n}$. Agent $i$ has an initial amount of money $m_{i}^{0} \in \mathbb{R}_{+}$and ends up, after trading, with an amount of money $m_{i} \in \mathbb{R}_{+}$. Agent $i$ aims at maximizing a utility function $u_{i}\left(m_{i}, x_{i}\right)$ over a set $\mathbb{R}_{+} \times U_{i}$ subject to the budget constraint

$$
m_{i}-m_{i}^{0}+\left\langle p, x_{i}-x_{i}^{0}\right\rangle \leq 0
$$

where the sets $U_{i} \subset \mathbb{R}^{n}$ are nonempty, closed and convex and the functions $u_{i}$ are continuously differentiable, concave and nondecreasing over $\mathbb{R}_{+} \times U_{i}$. In addition to the budget constraints (41) there are supply-demand requirements for money and goods, of the form

$$
\sum_{i=1}^{r}\left[m_{i}-m_{i}^{0}\right] \leq 0, \quad \sum_{i=1}^{r}\left[x_{i}-x_{i}^{0}\right] \leq 0 .
$$

The problem is to find an equilibrium value of the vector variable $(p, m, x)$ such that each utility function attains its maximum subject to the budget and the supply-demand constraints. It is shown in [12, Theorem 1] that under some mild conditions that are satisfied in the example displayed below an equilibrium always exists, moreover it satisfies a first-order optimality condition for each agent involving the Lagrange functions

$$
L_{i}\left(p, m_{i}, x_{i}, \lambda_{i}\right)=-u\left(m_{i}, x_{i}\right)+\lambda_{i}\left(m_{i}-m_{i}^{0}+\left\langle p, x_{i}-x_{i}^{0}\right\rangle\right)
$$

with a Lagrange multiplier $\lambda_{i} \geq 0, i=1, \ldots, r$, associated with the budget constraint (41). Adding the supply-demand constraints (42) written as complementarity conditions, we obtain a variational inequality for the vectors $p \in \mathbb{R}_{+}^{n}, m=\left(m_{1}, \ldots, m_{r}\right)^{\top} \in \mathbb{R}_{+}^{r}, x=\left(x_{1}, \ldots, x_{r}\right)^{\top} \in U_{1} \times U_{2} \times \ldots U_{r}$, and $\lambda=\left(\lambda_{0}, \ldots, \lambda_{r}\right)^{\top} \in \mathbb{R}_{+}^{r}$ of the form

$$
-g\left(p, m, x, \lambda, m^{0}, x^{0}\right) \in N_{C}(p, m, x, \lambda),
$$

where

$$
C=\mathbb{R}_{+}^{n} \times \mathbb{R}_{+}^{r} \times U_{1} \times \cdots \times U_{r} \times \mathbb{R}_{+}^{r},
$$

and

$$
g\left(p, m, x, \lambda, m^{0}, x^{0}\right)=\left(\begin{array}{c}
\sum_{i=1}^{r}\left[x_{i}^{0}-x_{i}\right] \\
\ldots \\
\lambda_{i}-\nabla_{m_{i}} u_{i}\left(m_{i}, x_{i}\right) \\
\ldots \\
\lambda_{i} p-\nabla_{x_{i}} u_{i}\left(m_{i}, x_{i}\right) \\
\cdots \\
m_{i}^{0}-m_{i}+\left\langle p, x_{i}^{0}-x_{i}\right\rangle \\
\cdots
\end{array}\right) .
$$

The initial endowments are represented by the vectors $m^{0}=\left(m_{1}^{0}, \ldots, m_{r}^{0}\right)^{\top} \in \mathbb{R}_{+}^{r}$ and $x^{0}=$ $\left(x_{1}^{0}, \ldots, x_{r}^{0}\right)^{\top} \in U_{1} \times U_{2} \times \ldots U_{r}$. In [12, Theorem 3] it is shown that the equilibrium mapping associated with (43) is strongly regular provided that for each agent $i$ the initial goods $x_{i}^{0}$ are sufficiently close to the equilibrium vector $\bar{x}_{i}$; in other words, when the trade starts with amounts 
of goods not too far from the equilibrium. Note that the first inequality in (42) does not appear in (43) since at equilibrium that automatically becomes an equality.

We consider a specific example where there are two agents with utility functions

$$
u_{i}\left(m_{i}, x_{i}\right)=\alpha_{i} \ln \left(m_{i}\right)+\beta_{i} \ln \left(x_{i}\right), \quad i=1,2,
$$

and a single good subject to the constraints

$$
x_{i} \in U_{i}=\left[\xi_{i}, \eta_{i}\right], \quad i=1,2
$$

for some positive $\xi_{i}$ and $\eta_{i}$. The variational inequality (43) for the vector $\left(p, m_{1}, m_{2}, x_{1}, x_{2}, \lambda_{1}, \lambda_{2}\right)$ has the following specific form:

$$
-\left(\begin{array}{c}
\sum_{i=1}^{2}\left[x_{i}^{0}-x_{i}\right] \\
\lambda_{1}-\frac{\alpha_{1}}{m_{1}} \\
\lambda_{2}-\frac{\alpha_{2}}{m_{2}} \\
\lambda_{1} p-\frac{\beta_{1}}{x_{1}} \\
\lambda_{2} p-\frac{\beta_{2}}{x_{2}} \\
m_{1}^{0}-m_{1}+\left\langle p, x_{1}^{0}-x_{1}\right\rangle \\
m_{2}^{0}-m_{2}+\left\langle p,\left[x_{2}^{0}-x_{2}\right\rangle\right.
\end{array}\right) \in\left(\begin{array}{c}
N_{\mathbb{R}_{+}}(p) \\
N_{\mathbb{R}_{+}}\left(m_{1}\right) \\
N_{\mathbb{R}_{+}}\left(m_{2}\right) \\
N_{U_{1}}\left(x_{1}\right) \\
N_{U_{2}}\left(x_{2}\right) \\
N_{\mathbb{R}_{+}}\left(\lambda_{1}\right) \\
N_{\mathbb{R}_{+}}\left(\lambda_{2}\right)
\end{array}\right) .
$$

The numerical implementation of Broyden's update (43) for this variational inequality has been done in Matlab. Each step of the method reduces to solving linear complementarity problems (LCP). The matlab function LCP by Yuval available at

http://www.mathworks.com/matlabcentral/fileexchange/20952 has been used for solving these problems. The computations are done for the following data. For the parameters $\alpha_{i}=\beta_{i}=0.1$ we consider the first agents with endowment of good 0.9 and money 1.3 and the second agent with unit endowments: $x^{0}=(0.9,1)^{\top}, m^{0}=(1.3,1)^{\top}$. The survival interval of consumption for each agent is $[0.94,1.08]$. Then the solution is: $p=1.2745, m=(1.2235,1.0765)^{\top}, x=(0.96,0.94)^{\top}$, $\lambda=(0.0817,0.0929)^{\top}$.

We did numerical testing with various starting points and starting updates, and obtained rather similar results. The result of one of these tests is presented below for the starting point of the algorithm equal $p_{0}=1.3745, m_{0}=(1.3235,1.1765)^{\top}, x_{0}=(1.06,1.04)^{\top}, \lambda_{0}=(0.1817,0.1929)^{\top}$ and initial update $B_{0}$ equal the value of the Jacobian at the starting point. The results of computations are given in Table 5.2. We have q-superlinear convergence also for this case, as proved theoretically.

\begin{tabular}{|c|c|c|c|}
\hline Iteration & $\left\|e_{k}\right\|$ & $\frac{\left\|e_{k+1}\right\|}{\left\|e_{k}\right\|}$ & $\frac{\left\|E_{k} s_{k}\right\|}{\left\|s_{k}\right\|}$ \\
\hline 1 & 0.26457513 & 0.20651049 & 0.03359845 \\
\hline 2 & 0.05463754 & 0.54349707 & 0.00097674 \\
\hline 3 & 0.02969534 & 0.00930219 & 0.00039065 \\
\hline 4 & 0.00027623 & 0.03250097 & 0.00000396 \\
\hline 5 & 0.00000898 & 0.01002655 & 0.00000021 \\
\hline 6 & 0.00000009 & 0.01152241 & 0.00000000 \\
\hline
\end{tabular}

Table 5.2. Numerical results for the second example.

Acknowledgment. The authors wish to thank the anonymous referees for their valuable comments and suggestions. 


\section{References}

[1] I. K. Argyros, Y. J. Cho, S. Hilout, Numerical methods for equations and its applications, CRC Press, Boca Raton, FL, 2012.

[2] H. H. Bauschke, P.L. Combettes, Convex Analysis and Monotone Operator Theory in Hilbert Spaces, Springer, 2011.

[3] B. Benahmed, H. Mokhtar-Kharroubi, B. Malafosse, A. Yassine, Quasi-Newton methods in infinite-dimensional spaces and application to matrix equations. Journal of Global Optim., 49 (2011), 365-379.

[4] J. F. Bonnans, Local analysis of Newton-type methods for variational inequalities and nonlinear programming, Appl. Math. Optim. 29 (1994), 161-186.

[5] J. E. Dennis, JR., J. J. Moré, A characterization of superlinear convergence and its application to quasi-Newton methods. Math. Comp. 28 (1974), 549-560.

[6] A. L. Dontchev, Characterizations of Lipschitz stability in optimization, in Recent Developments in Well-posed Variational Problems, pp. 96-116, Kluwer, Boston 1995.

[7] A. L. Dontchev, Local convergence of the Newton method for generalized equation. $C$. $R$. Acad. Sci. Paris, Sér. I 322 (1996), 327-331.

[8] A. L. Dontchev, Lipschitzian stability of Newton's method for variational inclusions, in System modelling and optimization, pp. 119-147, Kluwer, Boston 2000.

[9] A. L. Dontchev, Generalizations of the Dennis-Moré theorem, SIAM J. Optim. 22 (2012) $821-830$.

[10] A. L. Dontchev, W. W. Hager, An inverse mapping theorem for set-valued maps. Proc. Amer. Math. Soc. 121 (1994), 481-489.

[11] A. L. Dontchev, R. T. Rockafellar, Implicit functions and solution mappings. A view from variational analysis, Springer Mathematics Monographs, Dordrecht 2009.

[12] A. L. Dontchev, R. T. Rockafellar, Parametric stability of solutions in models of economic equilibrium. J. Convex Analysis 19 (2012), 975-997.

[13] A. Griewank, The local convergence of Broyden-like methods on Lipschitzian problems in Hilbert spaces. SIAM J. Numer. Anal. 24 (1987), 684-705.

[14] S. M. Grzegórski, Orthogonal projections on convex sets for Newton-like methods, SIAM J. Numer. Anal. 22 (1985) 1208-1219.

[15] D. M. Hwang, C. T. Kelley, Convergence of Broyden's method in Banach spaces. SIAM J. Optim. 2 (1992), 505-532. 
[16] N. H., Josephy, Newton's method for generalized equations and the PIES energy model, Ph.D. Dissertation, Department of Industrial Engineering, University of Wisconsin-Madison, 1979.

[17] C. T. KELlEy, Iterative methods for linear and nonlinear equations. With separately available software, Frontiers in Applied Mathematics 16 SIAM, Philadelphia, PA, 1995.

[18] C. T. Kelley, E. Sachs, A new proof of superlinear convergence for Broyden's method in Hilbert space. SIAM J. Optim. 1 (1991), 146-150.

[19] D. Klatte, B. Kummer, Nonsmooth Equations in Optimization, Kluwer 2002.

[20] R. Meise, D. Vogt, Introduction to Functional Analysis, Oxford University Press, 2004.

[21] S. M. Robinson, Strongly regular generalized equations. Math. Oper. Res. 5 (1980), 43-62.

[22] E. W. SACHS, Convergence rates of quasi-Newton algorithms for some nonsmooth optimization problems. SIAM J. Control Optim. 23 (1985), 401-418.

[23] E. W. SAchs, Broyden's method in Hilbert space. Math. Programming 35 (1986), 71-82.

[24] W. Sun, Y.-X. YuAn, Optimization theory and methods: nonlinear programming. Springer Optimization and Its Applications, 1. Springer, New York, 2006.

[25] Y. WEN-HUAN, A quasi-Newton method in infinite-dimensional spaces and its application for solving a parabolic inverse problem, J. of Comp. Math. 16 (1998), 305-318. 